\title{
YO! - A Time-of-Arrival Receiver for Removal of Femtosecond Helicity-Correlated Beam Effects"
}

\author{
J. Musson ${ }^{\dagger}$, T. Allison ${ }^{\dagger}$, A. Freyberger ${ }^{\dagger}$, J. Kuhn`, B. Quinn \\ ${ }^{\dagger}$ TJNAF, Newport News, VA, 23606, USA \\ Carnegie-Mellon University, Pittsburgh, PA, 15213, USA
}

\begin{abstract}
The G0 parity violation experiment at Jefferson Lab is based on time-of-flight measurements, and is sensitive to timing effects between the two electron helicity states of the beam. Photon counters triggered by time-of-arrival at the target mandate that timing must be independent of delays associated with different orbits taken by the two helicity states. In addition, the standard $499 \mathrm{MHz}$ beam structure is altered such that 1 of every 16 microbunches are filled, resulting in an arrival frequency of 31.1875 (31) $\mathrm{MHz}$, and an average current of $40 \mu \mathrm{A}$. Helicty correction involves identifying and tracking the 31 MHz subharmonic, applying a fast/fine phase correction, and finally producing a clean $31 \mathrm{MHz}$ trigger and a $499 \mathrm{MHz}$ clock train. These signals are phase-matched to the beam arrival at the target on the order of femtoseconds. The $10 \mathrm{kHz}$ output bandwidth is sufficiently greater than the $30 \mathrm{~Hz}$ helicity flip settling time $(500 \mu \mathrm{s})$. This permits the system to correct each helicity bin for any orbit-induced timing inequalities. A sampling phase detection scheme is used in order to eliminate the unavoidable $2 \Pi / \mathrm{n}$ phase shifts associated with frequency dividers. Conventional receiver architecture and DSP techniques are combined for maximum sensitivity, bandwidth, and flexibility. Results of bench tests, commissioning and production data will be presented.
\end{abstract}

\section{INTRODUCTION}

G0 is an experiment at Jefferson Lab designed to measure parity-violating asymmetries in elastic electron-nucleon scattering as a function of momentum transferred to the nucleon. This will provide better understanding of proton charge and magnetic structure by describing contributions from up, down and strange quarks [1].

At the heart of the experiment is JLAB's ability to deliver highly polarized $(\sim 75 \%)$ electrons, and to alternately "flip" the helicty from $+z$ to $-z$ at a $30 \mathrm{~Hz}$ rate using a pseudo-random code. It is possible that small helicity-correlated offsets associated with the launching time of the electron bunches could occur, on order of femtoseconds. This effect would produce a perceived asymmetry in the calculated time-of-flight of the particles from the target to the focal plane detectors.

The YO! Box produces two beam-derived signals, CLK and $\mathrm{Y}_{0}$, which are synchronized to the arrival of the electron bunches in experimental Hall $\mathrm{C}$, rather than to the laser-pulse associated with the time of launch in the Injector. In addition to tracking any time-of-flight variations, it is mandatory that the receiver be unaffected *This work was supported by the U.S. Department of Energy Contract Number DE-AC05-84-ER40150 
by variations in beam intensity and position. Also, RF sampling was chosen as an alternative to prescaling in an effort to remove the inevitable $2 \pi / \mathrm{n}$ phase ambiguity associated with frequency dividing. This ambiguity would create arbitrary cycle slips, catastrophically confusing the triggering of the photon detectors. Tight phase control and monitoring are also required to prevent cycle slips from within the control system.

The G0 beam is a modified $499 \mathrm{MHz}$ CEBAF bunch structure, whereby only every $16^{\text {th }}$ bucket of 2 ps duration contains electrons, but with 16 times the intensity. This results in a $31 \mathrm{MHz}$ sub-harmonic whose lower-limit level is nominally $-95 \mathrm{dBm}$ after redistributing the pulse-power energy and combining with the response from a standard stripline BPM designed for $1497 \mathrm{MHz}$ operation. Although the $\mathrm{S} / \mathrm{N}$ is too low for high precision timing, a filtered signal is easily derived for use as a sampling reference. Also available is energy from a resonant $1497 \mathrm{MHz} \mathrm{BCM}$ cavity, which is much greater $(-20 \mathrm{dBm})$ and affords good $\mathrm{S} / \mathrm{N}$ ratio for fine / fast tracking.

Timing is derived by phase-locking a stable $31.1875 \mathrm{MHz}$ VCXO to the $-95 \mathrm{dBm}$ $31.1875 \mathrm{MHz}$ sub-harmonic. The clean VCXO signal output is used to stroboscopically sample the $1497 \mathrm{MHz}$ beam-derived signal with a Sampling Phase Detector (SPD), establishing a high resolution (ps) zero crossing with $10 \mathrm{kHz}$ bandwidth. This phase correction is applied back to the $31 \mathrm{MHz}$ VCXO signal, defining the time-of-arrival. Finally, the corrected VCXO signal is again used to sample a Master Oscillator-derived (MO) $499 \mathrm{MHz}$ signal, applying the phase correction and aligning the 499 signal to create a CLK output. Figure 1 is an overview of the system.

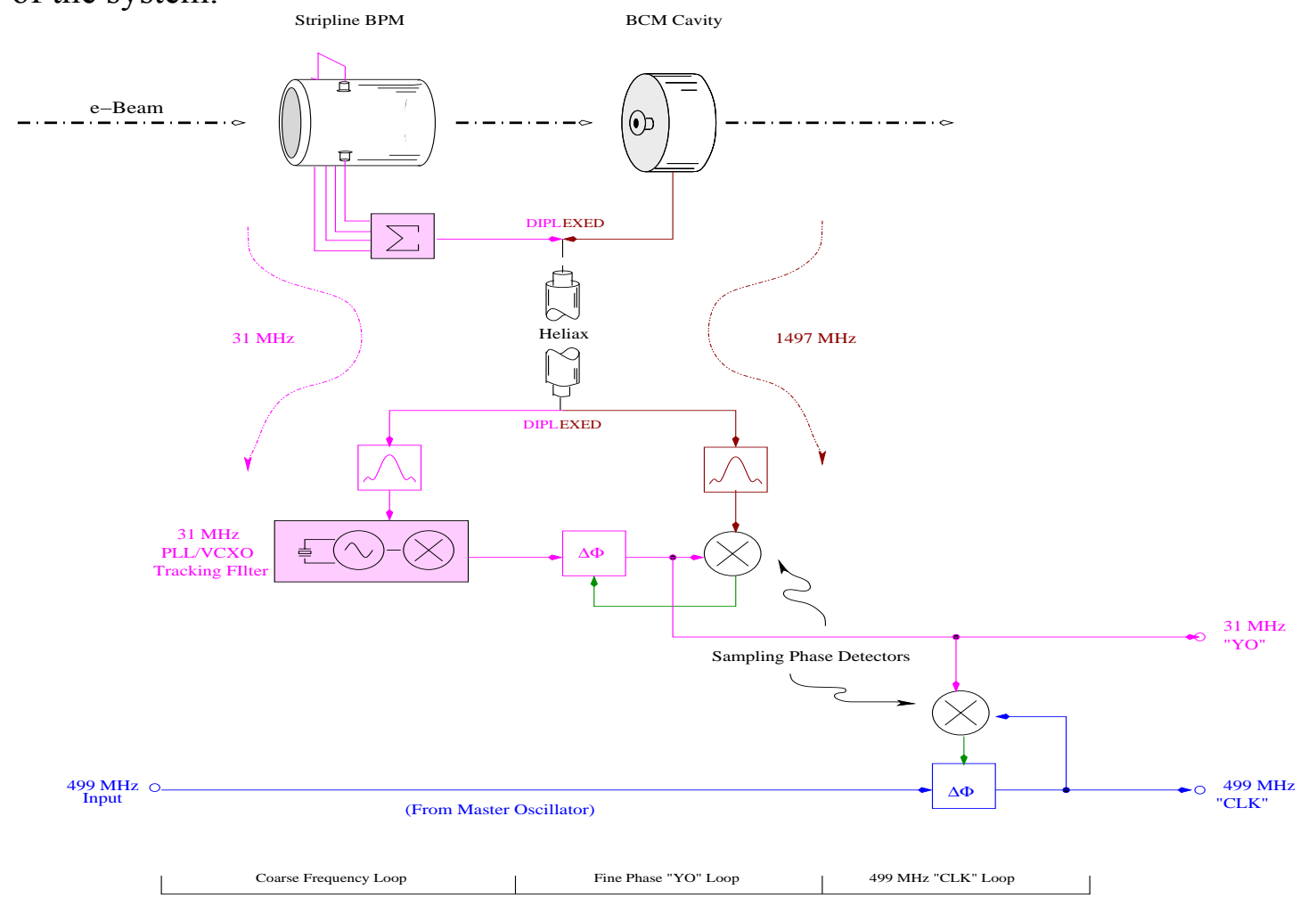

FIGURE 1. Receiver system overview. 


\section{RECEIVER ARCHITECTURE}

A hybrid analog / DSP architecture was selected primarily to piggy-back other development efforts at JLAB. Although the baseband FPGA-based DSP processor board was specifically designed for the YO! Receiver, it also resulted in a development tool for similar projects, and provided an ideal platform from which to develop the control algorithm. Due to the low noise figure requirement, combined with moderate output bandwidth, conventional RF front-ends were designed, but also included the use of Sampling Phase Detectors (SPD) for direct-conversion to baseband. Figure 2 is the block diagram of the entire receiver system.

In addition to bench testing sub-assemblies, extensive modeling using SystemView (Matlab) was performed in order to verify SPD operation, generate filter coefficients, and also check initial PID parameters for the control algorithm.

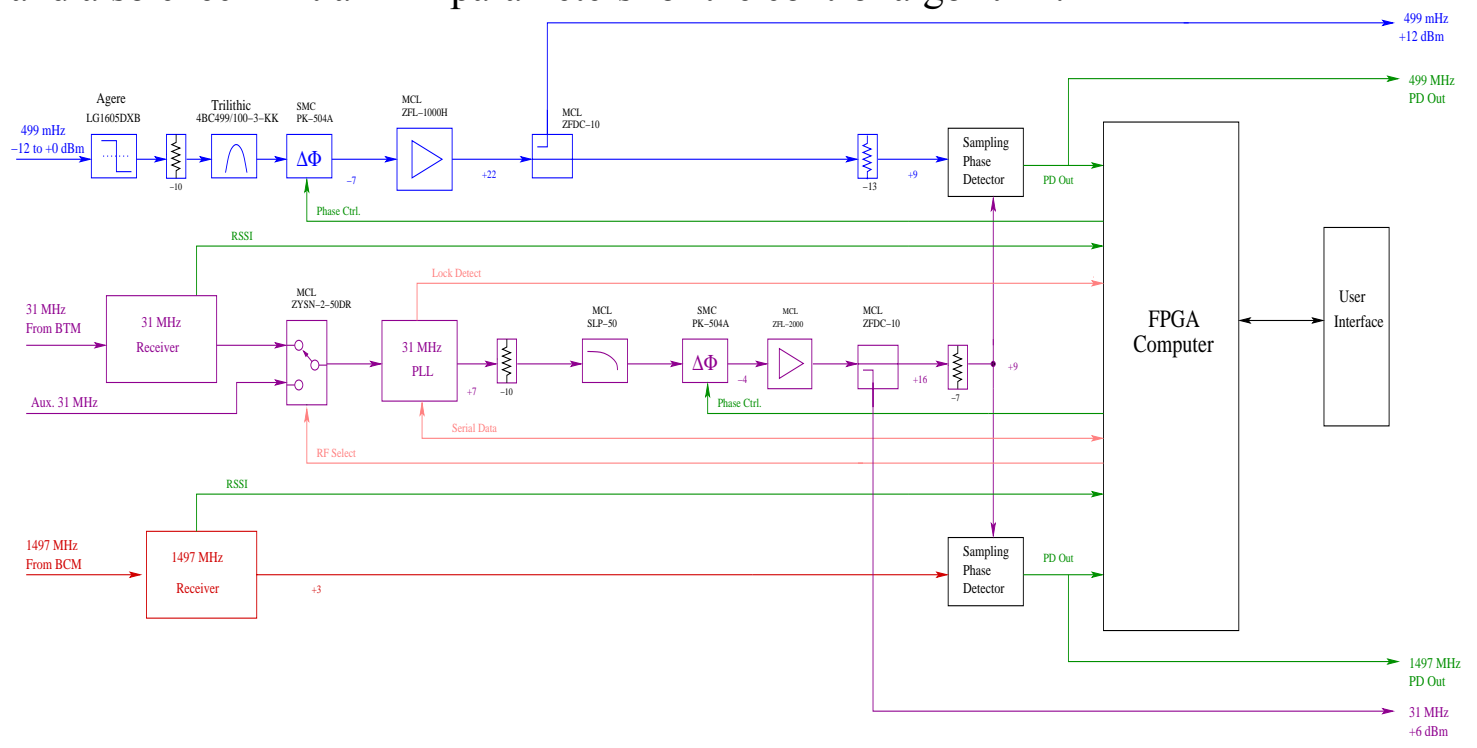

FIGURE 2. Receiver system block diagram

\section{SAMPLING PHASE DETECTOR (SPD)}

The fundamental advantage of the SPD, exploited by the YO! Receiver, is the ability to compare the phases of harmonically related signals, as opposed to conventional phase detectors which require identical frequency inputs. The concept of stroboscopically sampling a fast signal with an integral subharmonic has a rich and fascinating history [2]. References from as early as 1904 appear in literature [3], and were the basis for advancing vacuum tube technology to the point of observing microwave frequencies, resulting in affordable microwave instrumentation [4].

Although modern passive SPDs incorporating step-recovery diodes were initially investigated, they were found to be too lossy and fussy, and only moderate success was 
achievable in trial runs [5]. Ultimately, the selected SPD was based on a circuit which first appeared in a General Electric Transistor Manual in 1964 [6].NIST-based equivalent-time sampling circuits using comparators also provided promising results, but not at the intended operating microwave frequency $[7,8]$. A circuit using a PECL flip-flop and integrator was found which operates on the principle of balancing the $Q$ and /Q output durations by precisely holding the input at the logic decision threshold [9]. Feedback is used to balance the bias point at a metastable region, making the system highly sensitive to input perturbations. Input disturbances appear as pulsewidth modulation on the Q-outputs, which are low-pass filtered and integrated, appearing as a voltage after the integrator. Since PECL operates well into the microwave region, its speed was adequate for $1497 \mathrm{MHz}$ operation.

Since the sampling is synchronous, it possesses inherent noise immunity (much like a lock-in). In addition, the circuit is lossless, preserving the overall system noise figure [9].

A serious drawback to the single SPD is 2-quadrant operation, resulting in ambiguity between quadrants I/III and II/IV. This could either be resolved by dithering, or by using two SPDs in a quadrature configuration.

\section{DSP BASEBAND PROCESSOR}

Figure 3 illustrates the general functionality of the DSP Board. It consists of 4 analog-to-digital converter (ADC) stages, 4 digital-to-analog converter (DAC) stages, a FPGA, and multiple status and control inputs/outputs. The ADCs and DACs have a resolution of 16-bits and are updated at a rate of $150 \mathrm{kHz}$. Each ADC is preceded by an op-amp stage used for anti-alias filtering, scaling, and offsetting. The DAC outputs are also buffered with an op-amp stage used for scaling and filtering update-related noise. 


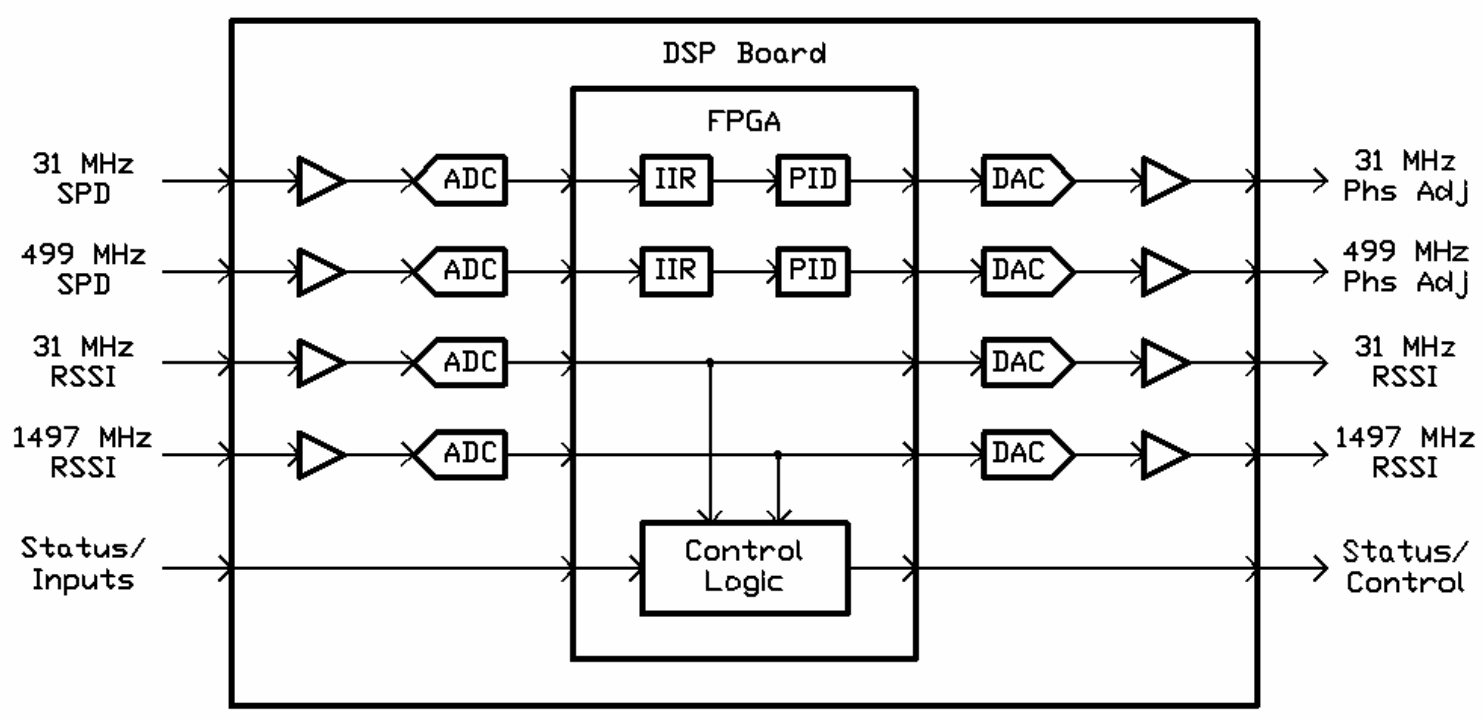

FIGURE 3. FPGA-based DSP processor

The digitized Sampling Phase Detector (SPD) signals are sent to the FPGA where they are first passed through an IIR filter then a PID algorithm. The IIR filters have a $3 \mathrm{~dB}$ roll off of $500 \mathrm{~Hz}$ and are implemented using equation (1). The filter constants $\left(a_{1}, b_{0}\right.$, and $\left.b_{1}\right)$ where derived using simulation software and scaled appropriately for this application. The variable $n$ represents a discrete sample time, $x(n)$ is the signal input, and $y(n)$ is the filter output.

$$
y(n)=a_{1} * y(n-1)+b_{0} * x(n)+b_{1} * x(n-1)
$$

The PID algorithm is accomplished with equations (2) and (3). The expression $e(n)$ is the filtered SPD error signal input, $S(n)$ is the integral term, and $u(n)$ is the PID output. The PID constants $\left(K_{P}, K_{I}\right.$, and $\left.K_{D}\right)$ can be selected with jumpers, updated using an optical encoder knob, and displayed via status LEDs. Once the control loop has been tuned, the associated PID constants are hard coded into the FPGA.

$$
\begin{aligned}
& u(n)=K_{P} * e(n)+K_{I} * S(n)+K_{D} *[e(n)-e(n-1)] \\
& S(n)=\frac{S(n-1)+[e(n)+e(n-1)]}{2}
\end{aligned}
$$

The control logic monitors the Received Signal Strength Indicators (RSSI) and the $31 \mathrm{MHz}$ Phase Adjust in order to intelligently switch the $31 \mathrm{MHz}$ input signal and establish lock. The RSSIs are used to determine if the signal levels are high enough to maintain lock. When the RSSIs become too low, the DSP Board freezes the IIR-PID$31 \mathrm{MHz}$ Phase Adjust output chain and switches the chassis to an external $31 \mathrm{MHz}$ reference. When the RSSIs return to an acceptable level, the chassis is switched back to the $31 \mathrm{MHz}$ input. The IIR-PID-31 MHz Phase Adjust chain remains frozen for $50 \mathrm{~ms}$ to permit the $31 \mathrm{MHz}$ input signal to propagate through the chassis and into the 
DSP board. This allows for seamless switching back-and-forth between the input signal and reference. If the chassis loses lock (the $31 \mathrm{MHz}$ Phase Adjust rails) the DSP Board automatically resets the IIR-PID-31 MHz phase adjust chain in an effort to re-establish lock. The Phase Adjust outputs are limited to discourage multiple lock points and to ensure that the same lock point is found each time. The control logic also provides various status outputs to indicate the state of the chassis.

\section{TESTS}

Along with component and sub-assembly testing, system performance tests were required as a condition of customer certification. Of these, static bench tests were performed which measured such parameters as phase detector scaling, residual offset voltages, sensitivities to varying beam currents and positions, and slow signal tracking. Dynamic measurements included open and closed-loop responses, output BW, loop damping / settling time, and tangential sensitivity to 31 and $1497 \mathrm{MHz}$. Finally, beambased tests were performed to provide the in-situ calibration data needed for accurate conversion from voltage data to engineering units.

Attempts to fully characterize the dynamic behavior of the YO! Receiver were performed using an HP 89410 Dynamic Signal Analyzer. The following stimulusresponse tests were performed by modulating a pre-characterized voltage-variable phase shifter to induce a known phase delay, verifying frequency response of the PID in closed-loop, as well as to determine tangential sensitivity at a $30 \mathrm{~Hz}$ modulation frequency (ultimately reported in fs). Figure 4 shows the measured results of the final $499 \mathrm{MHz}$ phase loop, confirming the well-behaved $10 \mathrm{kHz}$ loop BW (100 us response time) for the entire system. Figure 5 demonstrates $10 \mathrm{~dB} \mathrm{~S} / \mathrm{N}$ for a measurement of a 1 ps equivalent delay. Subsequent tests using lower phase noise RF sources produced tangential sensitivities at the $10 \mathrm{fs}$ level. All tests were performed with nominal $40 \mathrm{uA}$ simulated beam: $\mathrm{P}_{31}=-80 \mathrm{dBm}, \mathrm{P}_{1497}=-20 \mathrm{dBm}, \mathrm{S} / \mathrm{N}>=20 \mathrm{~dB}$.

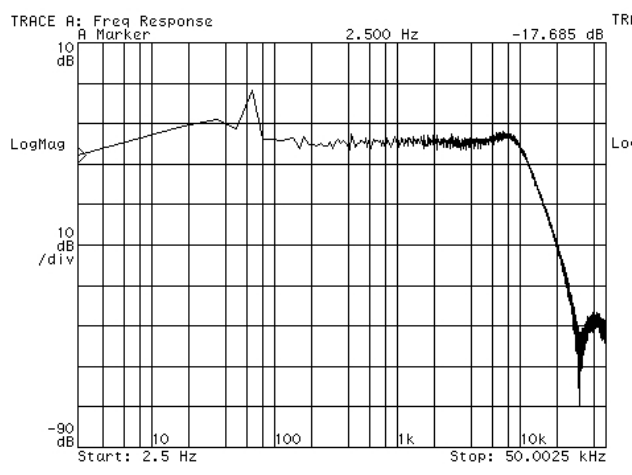

a.

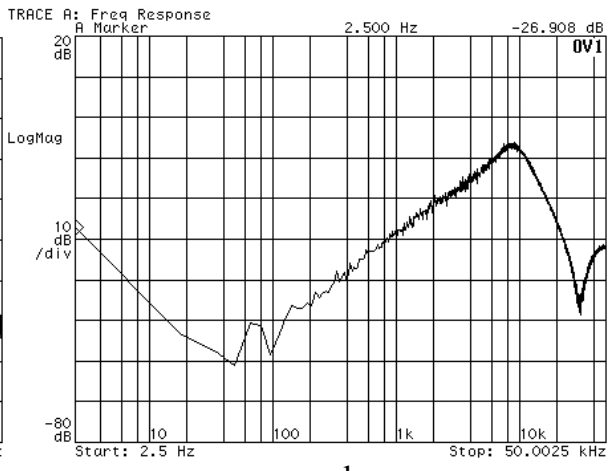

b.

FIGURE 4. Dynamic stimulus-response test plots of $499 \mathrm{MHz}$ phase-loop control (a) and error signals (b), performed using HP89410 Dynamic Signal Analyzer. 


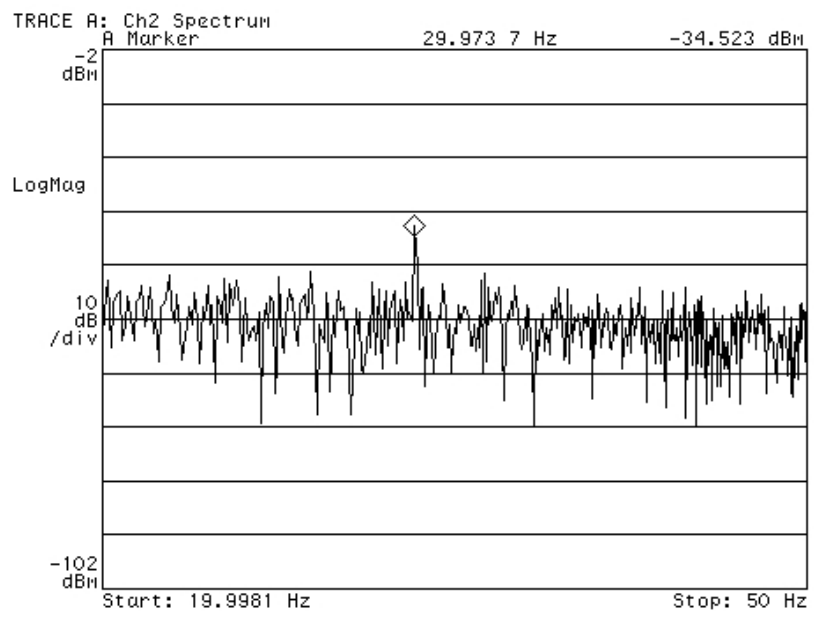

FIGURE 5. Measurement of $1 \mathrm{ps}$ equivalent phase delay at $30 \mathrm{~Hz}$ modulation, demonstrating $>10 \mathrm{~dB}$ $\mathrm{S} / \mathrm{N}$.

After installation in the experimental hall, test plans were executed, which verified the sensitivity as well as the proper scaling of the time-of-flight measurements. Since the relationship between magnet current and resulting path length within the accelerator is reasonably well known, the easiest method was to wiggle a dogleg magnet in the accelerator arc, intentionally modulating the path length by $+/-2$ ps. Data was gathered using a PC104-type single-board computer serving EPICS, and producing real-time statistics and FFTs. Figure 6 displays the measured delay (in ps) vs. dogleg magnet flux (g-cm).

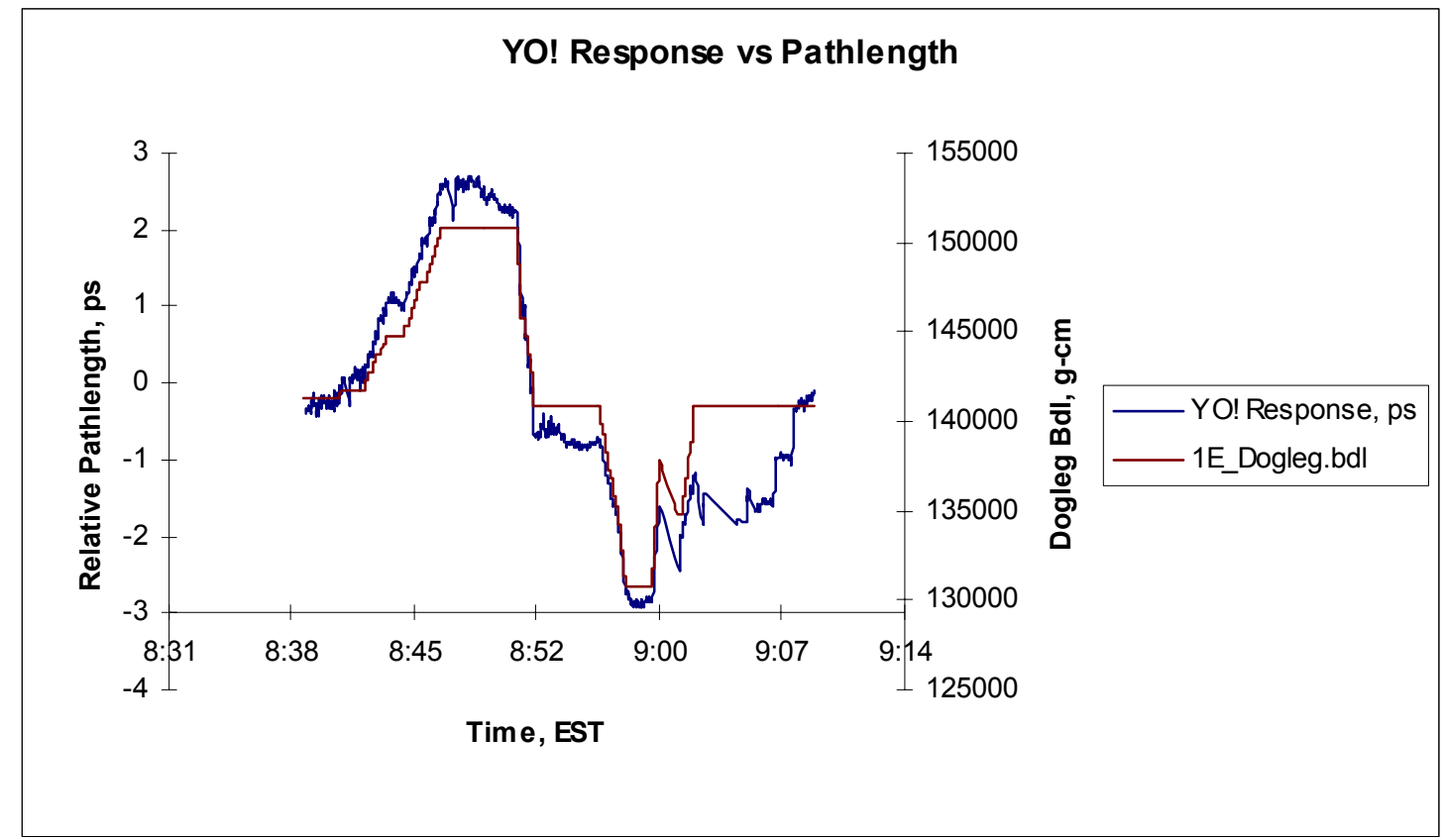

FIGURE 6. YO! Receiver output response of $499 \mathrm{MHz}$ phase control signal vs. Dogleg magnet setting. 
Although the G0 experiment was in-progress at the time of this writing, off-line data was analyzed at the $50 \%$ mark to verify proper operation. Of most interest were the immunities to current intensity fluctuations, as well as position. Figure 7 demonstrates both effects, resulting in certification of the receiver system by the G0 Collaboration [10].

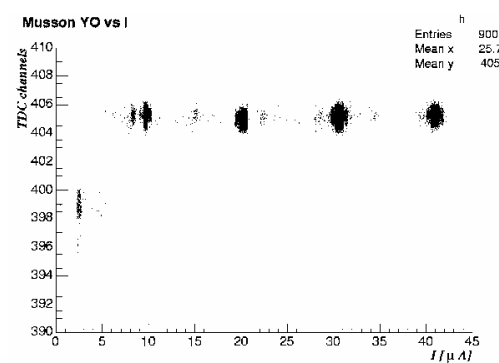

a.

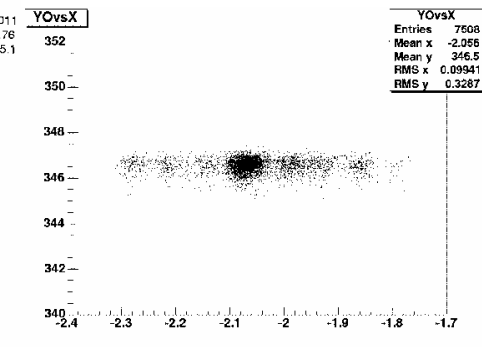

b.

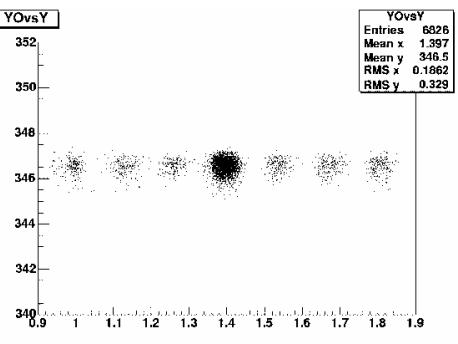

c.

FIGURE 7. Demonstration of immunities to beam intensity (a) and beam position for X (b) and Y (c). TDC bins each represent 35 ps.

\section{OTHER APPLICATIONS}

As is usually the case for new systems, measured data often describes an effect previously unmeasureable by existing hardware. The ability to measure pathlength at the end-station with high speed and resolution provides a feedback mechanism for energy locks, helicity control at the target, as well as corrections associated with seasonal machine growth. Finally, a multi-channel, multi-frequency vector voltmeter spinoff project is being produced for the purpose of Injector RF Phase monitoring, whereby an MO-derived $1497 \mathrm{MHz}$ signal is used as a reference to quadrature SPDs, providing ps-level resolution with $1 \mathrm{~Hz}$ update rates.

\section{ACKNOWLEDGEMENTS}

The authors would like to express their gratitude to the Jefferson Lab EES Group and CMU. Also to the Experimenters, with special thanks to Paul King for his assistance in YO! operations and data gathering, and Mark Pitt for his generous input on early design phases. 


\section{REFERENCES}

1. G0 Collaboration homepage, http://www.npl.uiuc.edu/exp/G0/G0Main.html

2. M. Kahrs, "50 Years of RF and Microwave Sampling," IEEE Trans. On Microwave Theory and Techniques, Vol. 51, No. 6, pp. 1787-1805 June 2003

3. E. Hospitalier, "The Slow Registration of Rapid Phenomena by Stroboscopic Methods," The

Electrical Engineer, pp. 40-44, Jan. 1904

4. M. Kahrs, "Applications of RF and Microwave Sampling to Instrumentation and Measurement,"

Proc. Of The 2003 International Microwave Symposium , Philadelphia, PA. Pages 1503-1506 (2003)

5. "Sampling Phase Detector Application Note," Metelics Corp.

http://www.metelics.com/sampling_phase_detector.htm

6. J.F. Cleary, GE Transistor Manual, $7^{\text {th }} \bar{E}$ d. (Circuits, Applications, Characteristics, Theory), p. 372

General Electric Co., Syracuse, NY, 1964

7. T.F. Souders, H.K. Schoenwetter, P.S. Hetrick, "Characterization of a Sampling Voltage Tracker for Measuring Fast, Repetitive Signals," IEEE Trans. On Instrumentation and Measurement, vol. IM-36, No. 4, December, 1987

8. O.B. Laug, M. Souders, D.R. Flach, "A Custom Integrated Circuit Comparator for High-Performance Sampling Applications," IEEE Trans. On Instrumentation and Measurement, vol. 41, No. 6, December, 1992

9. http://www3.sympatico.ca/add.automation (ADD Automation, Inc)

10. G0 Certification Document, "Results from the G0 Engineering Runs (Answers to the Certification Questions)," Submitted to JLAB March 5, 2004. 